\title{
Design and characterization of high-Q SAW resonators based on the AlN/Sapphire structure intended for high-temperature wireless sensor applications
}

\author{
Jeremy Streque, Julien Camus, Thierry Laroche, Sami Hage-Ali, Member, IEEE, Hamid M'Jahed, \\ Mohammad Rammal, Thierry Aubert, Mohamed Abdou Djouadi, Sylvain Ballandras, and Omar \\ Elmazria, Member, IEEE
}

\begin{abstract}
Aluminium nitride piezoelectric thin films grown on sapphire are strong candidates for high-temperature surface acoustic wave (SAW) sensors, due to their thermal stability, large bandgap, high acoustic velocity and suitable electromechanical coupling. However, thin-film resonators need more design efforts than those based on bulk crystals, due to the usually limited thickness of the piezoelectric films, and to acoustic properties disparities between the latters and their host substrate. This work presents an optimization of AIN/Sapphire-based SAW resonators with high quality factors for high-temperature applications. It combines specifically grown, $3 \mu \mathrm{m}$-thick aluminium nitride films, with the use of aluminium electrodes for their low density and resistivity, as an alternative to heavier electrodes like Pt. These electrodes allow for much lower mechanical losses and higher quality factors, in spite of needing passivation for increased lifetime. A standard resonator design is first presented and used for preliminary tests, in order to monitor the AIN/Sapphire structure with unprotected aluminium electrodes, for temperatures up to $600^{\circ} \mathrm{C}$. A quasi-synchronous, optimized design is then proposed for higher quality factors and wireless sensing compliance. The high temperature characterizations confirmed that much larger quality factors can be retrieved from this optimized design. The quasi-synchronous resonators proposed in this study remain well-tuned for temperatures up to $400^{\circ} \mathrm{C}$, and show high quality factors, as high as 3400 at $400^{\circ} \mathrm{C}$.
\end{abstract}

Index Terms-Sensors, Surface acoustic waves, Resonators, Aluminium nitride, High temperature.

\section{INTRODUCTION}

$\mathrm{S}$ URFACE acoustic wave (SAW) devices have been strongly promoted for their sensing capabilities on a wide range of physical quantities [1]. This includes - among others temperature, pressure, and strain sensors, that are naturally compatible with batteryless and wireless operation, making this technology particularly suitable for high-temperature

This work was supported by the French National Research Agency (project "SALSA", under the reference: ANR-15-CE08-0015-05) and in part by the French PIA project "Lorraine Université d'Excellence" under Grant ANR-15IDEX-04-LUE.

J. Streque is the corresponding author (e-mail: jeremy.streque@gmail.com).

J. Streque, S. Hage-Ali, H. M'Jahed, and O. Elmazria are with Institut Jean Lamour (IJL) UMR 7198, Université de Lorraine - CNRS, Nancy, France. applications. However, the availability of piezoelectric materials with good physico-chemical stability proves to be crucial in this context. The usual SAW substrates such as quartz and congruent lithium niobate are not considered as suitable for high-temperature operation above $400^{\circ} \mathrm{C}[2,3]$. So far, langasite $\left(\mathrm{La}_{3} \mathrm{Ga}_{5} \mathrm{SiO}_{14}\right.$; LGS) remains the only bulk material whose stability in air atmosphere has been demonstrated for long lifetimes, up to $1000^{\circ} \mathrm{C}$ [4]. However, its relatively low SAW velocity (2500-3000 m.s $\mathrm{s}^{-1}$ ) and high acoustic propagation losses are drawbacks for high frequencies, while its electrical resistivity drops with temperature due to oxygen ion transport and diffusion in the lattice, making it difficult to design resonators operable above $700^{\circ} \mathrm{C}$ [5]. In the last decade, aluminum nitride (AlN) piezoelectric thin films grown on sapphire substrates have been proved to be strong candidates as an alternative to LGS for high-temperature SAW applications due to their thermal and chemical stability up to more than $1000^{\circ} \mathrm{C}$ [6-8]. This bilayer structure offers moderate electromechanical coupling coefficient, comparable to that of LGS crystals (0.3-0.4\% at best), suitable for the achievement of SAW resonators. On the other hand, the AlN/Sapphire structure is compatible with high-frequency applications due to its large acoustic velocity (around $5500 \mathrm{~m} / \mathrm{s}$ ). Its main advantage for the intended application is likely the huge bandgap of AlN $(6.2 \mathrm{eV})$ which results in outstanding dielectric properties at high temperatures up to $1000^{\circ} \mathrm{C}$ and more [5]. The suitability of the AlN/Sapphire bilayer structure for high-frequency hightemperature SAW applications has thus been established. However, several challenges remain to be faced before commercializing SAW sensors based on this structure. In particular, SAW resonators with quality factors of several thousands, compatible with wireless interrogation, are highly needed. Thin-film, layered, resonators need more design efforts than those based on bulk crystals. It is also necessary to pay

J. Camus and M. A. Djouadi are with Institut des Matériaux Jean Rouxel IMN, UMR 6502, Université de Nantes, Nantes, France.

T. Laroche and S. Ballandras are withFrec|n|sys SASU - Besançon, France. T. Aubert is with CentraleSupélec - Université de Lorraine - Metz, France. 
attention to the evolution of the Q-factor of the resonators with the temperature. This evolution has not been reported so far. Therefore, this work presents an optimization of AIN/Sapphire based SAW resonators for high quality factors $(\mathrm{Q})$ and the evolution of this parameter with temperature, up to $400^{\circ} \mathrm{C}$.

\section{Considerations on Resonator Design AND OPTIMIZATION}

This study focuses on the $433 \mathrm{MHz}$ and $868 \mathrm{MHz}$ industrial, scientific and medical (ISM) bands : the typical wavelengths associated to these operation frequencies are respectively around 13 and $6.5 \mu \mathrm{m}$ on the studied AlN/Sapphire structure. The modelling was performed on a custom FEM/BEM software, including harmonic admittance calculations, with no electrical or mechanical loss considered. An introduction to the techniques used in the software can be found in refs. [9-11].

Initial simulations have been performed in order to assess the performance of resonators as a function of electrode thickness for a variety of electrode materials showing different densities, including Pt and Al (Figure 1), as a function of metallization ratio and relative electrode thickness $\left(h_{e l} / \lambda\right)$. The use of high-density electrode materials, like platinum or iridium-based alloys, leads to a strong decrease in the intrinsic
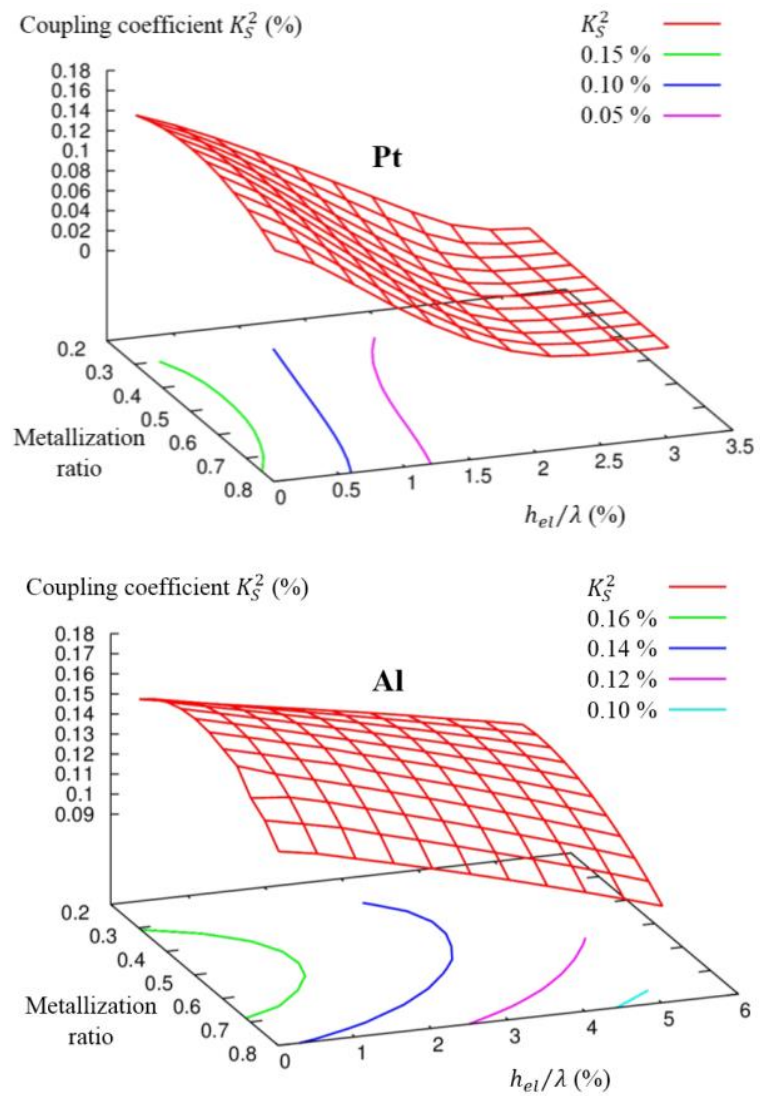

Fig. 1. Expected coupling coefficient for AlN/Sapphire structures under infinite electrode gratings (top: $\mathrm{Pt}$, left: $\mathrm{Al}$ ). Calculations made for an $\mathrm{AlN}$ thickness of $3 \mu \mathrm{m}$

electromechanical coupling coefficient $\left(K_{S}^{2}\right)$ of the substrate. Values of $K_{S}^{2}$ are typically twice lower for platinum than for lighter electrodes like aluminium, for an electrode relative thickness of $1 \%$, and nearly 20 times lower for $h_{e l} / \lambda=2 \%$. Thus, in the case of Pt IDTs, $K_{S}^{2}$ is below $0.03 \%$ for an electrode relative thickness of $2 \%$, and only $0.07 \%$ if this ratio is decreased to $1 \%$. At the same time, the lower conductivity and higher mechanical damping in Pt will generally lead to lower Q compared to Al. Lower $K_{S}^{2}$ and Q would lead to low figures of merit: this would have a large negative impact on the wireless capabilities of such devices, reducing the interrogation distance to only some tens of centimeters at best. Moreover, the use of thinner Pt electrodes would intensify their coalescence encountered at high temperature, and thus obliterate the advantages of such electrode material.

Moreover, this study proposes to improve the quality factor by introducing a detuning between the interdigitated fingers (IDTs) and the reflectors, thus providing a quasi-synchronous resonator design. The resulting effective coupling coefficient is thus expected to decrease: this definitively invalidates the use of heavy electrodes for this application. Aluminium, on the contrary, shows a lower thermal stability compared to these heavy electrodes, but is still worthy of attention, especially when passivation or packaging can address its oxidation issues [12]. The choice of aluminium electrodes was then made for their low density and resistivity, as an alternative to heavier electrodes like Pt, in spite of their lower thermal stability.

In a first time, standard, synchronous resonators operating around the $433 \mathrm{MHz}$ and $868 \mathrm{MHz}$ (ISM) bands are investigated. Based on these characterizations, a quasisynchronous, optimized design is then proposed for higher quality factors and wireless sensing compliance. Nonsynchronous designs have been previously presented in the Literature for various resonator structures $[13,14]$. The slight detuning that results from non-synchronicity between the IDTs and reflectors, allows to reshape the resonance spectrum of the devices, and thus offers larger quality factors which is an essential parameter for the remote interrogation of SAW resonators.

The optimized AlN thickness was determined from the $K_{S}^{2}$ dispersion curves in order to keep a near-maximal electromechanical coupling at $433 \mathrm{MHz}$ : it was chosen to be $3 \mu \mathrm{m}$. These modelling results are in good accordance with the studies previously reported in the Literature [15]. The targeted AlN thickness is beyond the usual thicknesses proposed by AlN films providers: a specific process has then been developed for the growth of thick AlN.

\section{EXPERIMENTAL RESULTS}

\section{A. Substrate Preparation}

The growth of $3 \mu \mathrm{m}$ thick AlN substrates was performed on the basis of $25 \mathrm{~nm}$ thick AlN/Sapphire buffer templates wafers provided by Kyma Technologies, Rayleigh, NC, USA, and based on an hydride vapor phase process. The piezoelectric layers were then deposited by DC magnetron sputtering (DCMS). Pinnacle ${ }^{\mathrm{TM}}$ Plus $5 \mathrm{KW}$ with arc-suppression features was used. Power supply parameters and Deposition conditions were optimized to obtain efficient spark suppression. The use 
of these low thickness AlN templates proved to offer a better crystalline quality, compared to the direct AlN deposition by DCMS on bare sapphire substrates. The deposition process was optimized in order to preserve the high crystalline quality of the AlN buffer layers for a thickness up to $3 \mu \mathrm{m}$, with near-epitaxial quality, leading to the expected structure for study: AlN (0001) / sapphire (0001) samples [16,17]. DCMS was chosen over metalorganic chemical vapor deposition (MOCVD) and molecular beam epitaxy (MBE) techniques [18], due its ability for strain management, which is crucial when it comes to achieve $3 \mu \mathrm{m}$ thick layers. It was also preferred to high power impulse magnetron sputtering, because of the higher strains encountered with this latter sputtering technique $[19,20]$. In order to keep residual strain as low as possible, the pressure was set to $2.2 \mathrm{mTorr}$, and the power to $23 \mathrm{~W} / \mathrm{cm}^{2}$ of target. The deposition rate then reached $130 \mathrm{~nm} / \mathrm{min}$. The thickness of the deposited AlN layers was then determined by ellipsometry, following a Tauc-Lorentz model.

The synthesized AlN films are highly textured despite their large thickness, and show typical XRD FWHM rocking-curve values of $0.8^{\circ}$ for the (002) reflex. This value remains close to the FWHM of the original $25 \mathrm{~nm}$ thick seed layer of AlN present on the templates, which was measured at $0.5^{\circ}$, as shown in Figure 2. It is also in good accordance with the properties of other thick AIN layers previously reported in the Literature by Martin et al. [21], who stated that the rocking curves tend to tighten with AlN thickness (reporting $1.14^{\circ}$ for $2 \mu \mathrm{m}$ thick films). This indicates an overall preservation of the AlN crystallinity throughout its deposition by DCMS, with a very limited rise of its mosaicity.



Fig. 2. XRD rocking curve of $3 \mu \mathrm{m}$ thick AlN for (0002) peak, with respect to $25 \mathrm{~nm}$ AlN templates for comparison. Insert: AFM mapping of a 10x10 $\mu \mathrm{m}^{2}$ of the same PVD grown AlN layer.

The resulting roughness of these 3- $\mu \mathrm{m}$ thick AlN layers was also measured by atomic force microscopy (AFM), leading to a typical RMS roughness of $30 \mathrm{~nm}$ (Figure 2: insert). However, this level of roughness did not interfere with the further processing of the AlN layers.

In addition, $\varphi$-mode XRD scans were used to study the epitaxial quality of the AlN films, as shown in Figure 3. These give access to the in-plane texturing of the deposited layer. While the column twist of (1011) planes was initially measured at $1.3^{\circ}$ for the $25 \mathrm{~nm}$ thick AlN templates used in the study, the further deposition of $3 \mu \mathrm{m}$ thick AlN led to a very limited overall column twist of $1.8^{\circ}$, demonstrating the quasiepitaxial nature of the grown films.

\section{B. Fabrication and Characterization of Standard Resonators}

A first series of SAW devices was then fabricated on AlN/Sapphire substrates. The $200 \mathrm{~nm}$-thick electrodes were deposited by DCMS. As mentioned hereinabove, the first series of devices, used to extract the material parameters for design optimization purposes, were based on synchronous resonators ( $\lambda=6.5 \mu \mathrm{m}$, aperture $=40 \lambda, 100$ pairs, $2 \times 200$ reflectors). The IDT patterns were defined by UV lithography, and electrodes were etched using a mix of orthophosphoric and nitric acids.

The experimental tests were performed under air atmosphere, using an Agilent N5230A network analyzer and a RF probe station (S-1160, Signatone Corp., Gilroy, CA), equipped with a thermal probing system up to $600^{\circ} \mathrm{C}$ (S-1060, Signatone). The $\mathrm{RF}$ probes were adapted in order to withstand such high temperature measurements.

Thus, the resonance frequencies, $Q$ factors and effective coupling coefficients could be extracted from the measurements of the resonators' admittances over this temperature range. $Q$ was first obtained from the plotting of the real part of the resonator admittance $Y_{11}$, and expressed as the ratio, noted $Q_{Y}$,

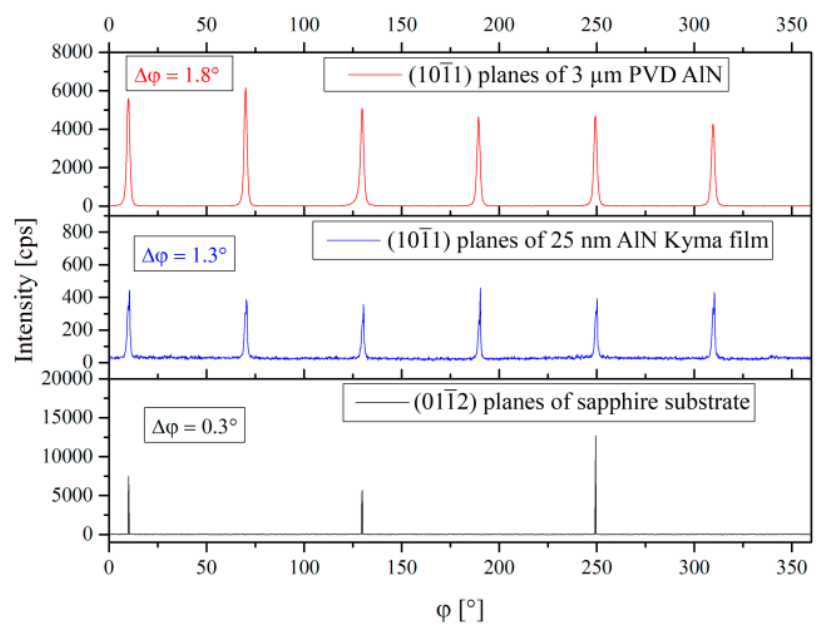

Fig. 3. X-ray diffractogram in $\varphi$-scan mode of $3-\mu \mathrm{m}$ thick AlN obtained by PVD, and deposited on AlN Kyma (25 nm)/Sapphire.

of its peak frequency to its FWHM [18]. Another method was also applied for the optimized resonators, through the study of the phase shift occurring at the parallel $\left(f_{p}\right)$ and series $\left(f_{s}\right)$ resonance frequencies, as shown by the following formula proposed by Lakin $[22,23]$ :

$$
Q_{s, p}=\frac{f_{s, p}}{2}\left|\frac{d \varphi_{Z}}{d f}\right|,
$$


Where $\varphi_{Z}$ is the phase of the impedance $Z_{11}$ of the resonator. Both methods are accepted among others [20], as being as valid for the retrieval of $Q$. The Lakin method allows to dissociate the parallel and series quality factors, that are related to electrical and mechanical losses, according to the Butterworth-van Dyke (BVD) model.

The effective coupling coefficient was also determined with the following formula:

$$
k_{e f f}^{2}=1-\frac{f_{s}{ }^{2}}{f_{p}{ }^{2}}
$$

The characterizations of standard resonators $(\lambda=6.5 \mu \mathrm{m})$. show typical values of $Q=325$ and $k_{\text {eff }}^{2}=0.56 \%$ at room temperature (Figure 4), for a relative AIN thickness of 0.46 .

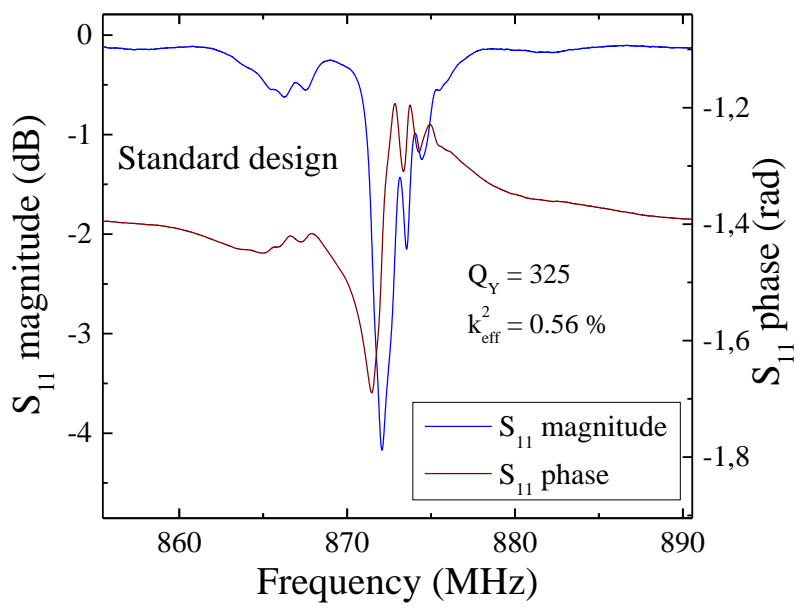

Fig. 4. Room temperature characterization of standard SAW resonators $(\lambda=6.5$ $\mu \mathrm{m})$, exhibiting spurious peaks in its return loss spectrum, and poor compatibility with wireless interrogation requirements., due to its low quality factor of 325 .

The standard resonators performed very well in high temperature characterizations. Their frequency evolution up to $593^{\circ} \mathrm{C}$ has a slight curvature, with a first-order TCF of -28 $\mathrm{ppm} /{ }^{\circ} \mathrm{C}$, and a second-order TCF of $-36 \mathrm{ppb} /{ }^{\circ} \mathrm{C}^{2}\left(25^{\circ} \mathrm{C}\right.$ being used as the reference temperature). This frequency behavior is in good accordance with previously published, experimental and calculated TCF results, for a relative AlN thickness around 0.46 [25]. The aluminium IDTs can still show enough stability in their electrical behavior up to $600^{\circ} \mathrm{C}$. The $Q$ factor of these standard resonators based on $\mathrm{Al} / \mathrm{AlN} / \mathrm{Sapphire}$ structure also remains almost constant, but at a value poorly compatible with wireless sensing, except at close distance.

\section{Fabrication and Characterization of Optimized Resonators}

The data extracted from the room temperature and high temperature characterizations of these standard resonators was then used to define a new, optimized resonator design, in order to reach higher $Q$ factors, and thus better wireless sensing capabilities. The new design is based on a one-port quasi- synchronous resonator design [26]. The geometrical parameters of the IDTs and of the reflectors were defined to keep the $S_{11}$ peak frequency close to the higher limit of their frequency bandpass. The corresponding wavelengths were respectively equal to $\lambda_{I D T}=12.64 \mu \mathrm{m}$, and to $\lambda_{\text {refl }}=12.57 \mu \mathrm{m}$. The resonator design was then adjusted to maximize the electromechanical coupling through its parallel resonance frequency, which was positioned close to the impedance matching frequency of the resonator. The numerical evaluation of its quality factor is 8500 .

This new resonator design was then process by UV lithography, using this time lift-off for the definition of the 200 $\mathrm{nm}$ thick aluminium electrodes.

Room temperature measurements were first performed on this series of optimized resonators. As expected, the optimized design shows a much better impedance matching, with return losses over $-15 \mathrm{~dB}$ and $Q$ values up to 8140 , and a smaller $k_{\text {eff }}^{2}$ : $0.031 \%$, as shown in Figure 5. The quality factor values were also retrieved from the impedance phase, for both parallel and series resonances. The series quality factor, illustrating mainly the mechanical losses, was calculated as $Q_{s}=6380$, while the parallel quality factor, that also takes into account the electrical losses, was determined as $Q_{p}=6300$.

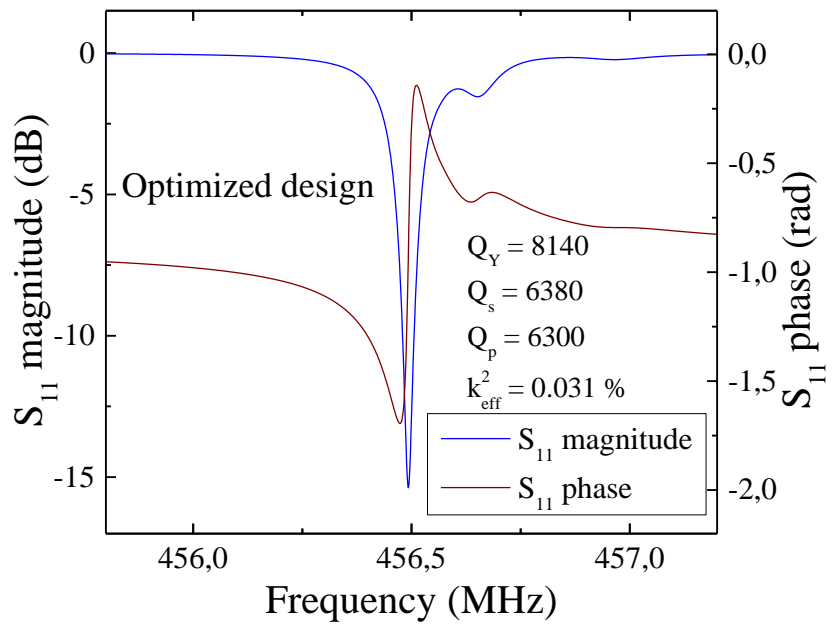

Fig. 5. Room temperature characterization of optimized quasi-synchronous SAW resonators $\left(\lambda_{I D T}=12.64 \mu \mathrm{m}, \lambda_{\text {refl }}=12.57 \mu \mathrm{m}\right)$, exhibiting much better quality factor (8140) and cleaner $S_{11}$ spectrum.

The resonators central frequency was noticed to be slightly shifted outside the $433 \mathrm{MHz}$ band, which can be easily corrected through a new design. The figure of merit obtained by the product of $Q$ and $k_{\text {eff }}^{2}$ is also improved by $38 \%$ through this optimization process, and also proves the usability of such structures for wireless operation [27].

The resonators were then individually characterized at higher temperature, and the RF signals $-S_{11}$ and admittance magnitudes - were also retrieved for each temperature plateau, after complete stabilization of the chuck temperature. These spectra are much well defined than with the previously studied standard resonators. The admittance spectra extracted from high temperature characterizations (Figure 6) show clear 
parallel and series resonance, in spite of a moderate increase of their impedance mismatch with temperature. A complete recovery of the signals happens during the cooling phase, as long as the temperature does not exceed $400^{\circ} \mathrm{C}$.

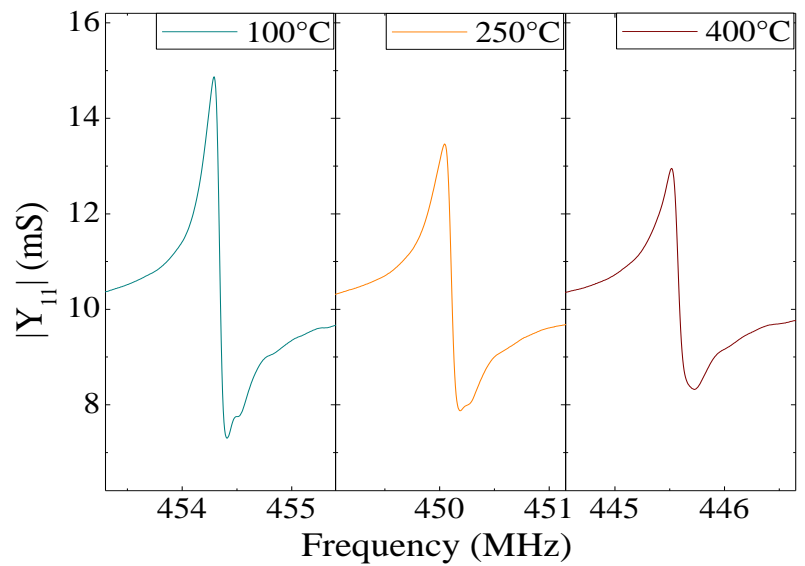

Fig. 6. Admittance spectra extracted from high-temperature characterization of optimized resonators $\left(\lambda_{\mathrm{IDT}}=12.64 \mu \mathrm{m}\right)$. Spectra show very clear parallel and series resonances, in spite of a moderate increase in impedance mismatch with temperature.

When heated up to $450^{\circ} \mathrm{C}$, the resonators can still sustain RF measurements, but at the cost of a decrease of the signal. Contrary to previous cycles up to $400^{\circ} \mathrm{C}$, the initial performance of the optimized resonators could not be recovered after cooling. It can be explained by the behavior of the $\mathrm{Al}$ electrodes: they require passivation for proper operation with this optimized design, which is much more sensitive to the electrical behavior of the electrodes. It then results in a progressive impedance mismatch, that explains the observed signal loss at temperatures higher than $450^{\circ} \mathrm{C}$, compared to the results obtained on the standard resonator design. However, the characterization of the optimized resonators for cycles up to $400^{\circ} \mathrm{C}$ leads to a clean, more linear TCF behavior, with a low drift through the successive heating and cooling cycles (Figure 7). The first-order TCF is $-57 \mathrm{ppm} /{ }^{\circ} \mathrm{C}$, while the second-order TCF is $-17 \mathrm{ppb} /{ }^{\circ} \mathrm{C}^{2}$. This is again in good agreement with previous results, for a relative AlN thickness of 0.24 [25]. The temperature reading errors are particularly low, in spite of the limited resolution of the chuck's temperature display $\left(1^{\circ} \mathrm{C}\right)$, that served as a reference.

A further analysis of the high temperature spectra has been performed, in order to retrieve the quality factor and the effective coupling coefficient as a function of temperature (Figure 8). The quality factor, while decreasing with temperature, remained high up to $400^{\circ} \mathrm{C}$, and sustained several heating cycles up to this temperature. At $400^{\circ} \mathrm{C}$, it is still at $50 \%$ of its room temperature value, which is well enough for proper wireless operation. The effective coupling coefficient follows an inverse trend, with a regular increase with temperature. Overall, the $Q . k_{e f f}^{2}$ maintains well through the whole temperature cycles, which goes along with the expectedly good preservation of the piezoelectric properties of the AlN layer at high temperatures. However, higher temperatures tend to strongly decrease the performance of the optimized resonators.
When the temperature reached $450^{\circ} \mathrm{C}$, a stronger decrease of the quality factor was noticed, down to 2900 .

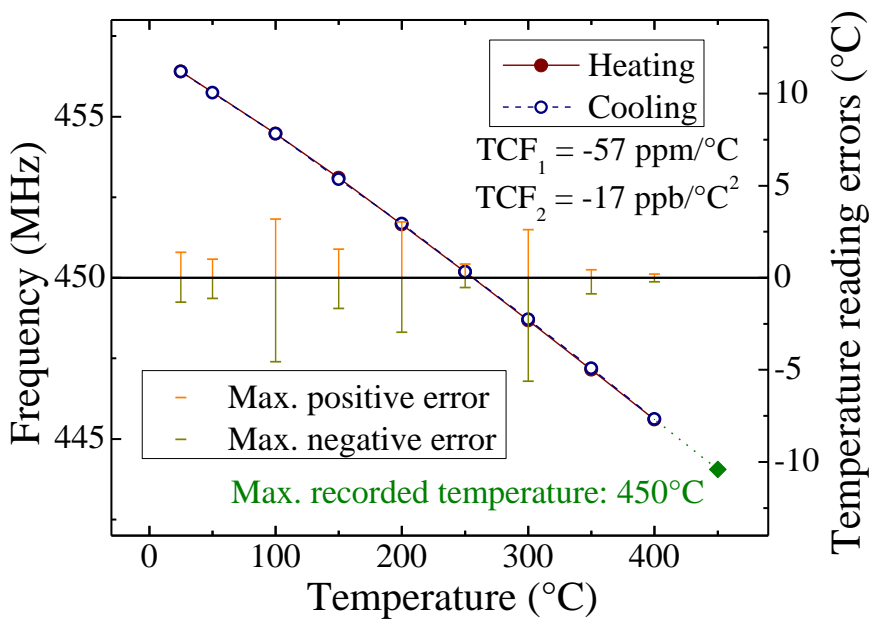

Fig. 7. TCF curve and temperature reading errors of an optimized resonator $\left(\lambda_{\text {IDT }}=12.64 \mu \mathrm{m}\right)$, showing very good reversibility and repeatability in temperature reading up to $400^{\circ} \mathrm{C}$. Signal loss happens when temperature is increased and maintained to $450^{\circ} \mathrm{C}$.

A variant of these optimized resonators, with higher metallization ratio $(\mathrm{MR}=60 \%)$, was also tested. Their quality factor was expectedly lower (4820 at room temperature), and their coupling coefficient higher $(0.051 \%)$. The resulting figure of merit was similar at room temperature, and nearly constant up to $400^{\circ} \mathrm{C}$ (as shown in Figure 8, for comparison purposes). Their better overall stability can be explained by the lesser resistance of their larger IDT fingers, which indicates that a proper passivation of the electrodes could bring more stability at high temperature.

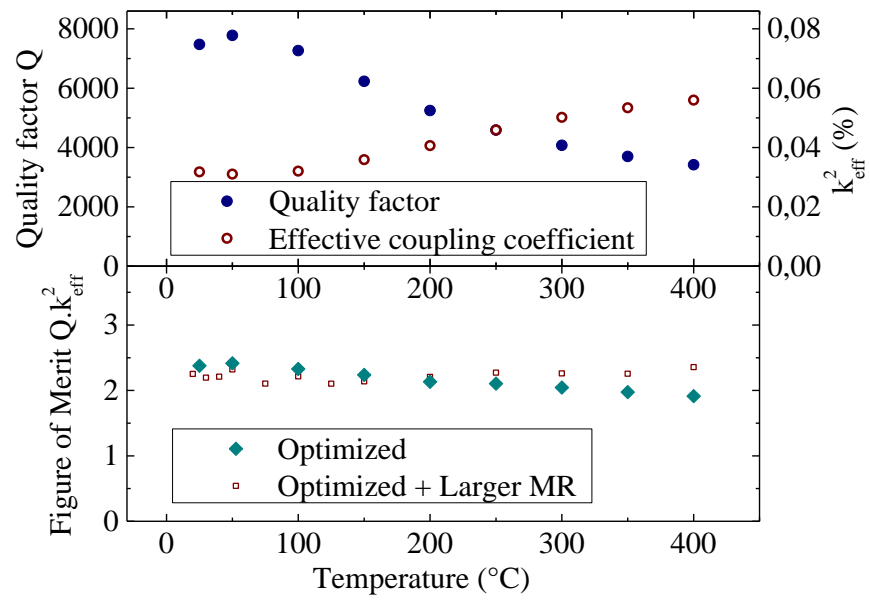

Fig. 8. Room temperature characterization of optimized quasi-synchronous SAW resonators $\left(\lambda_{\mathrm{IDT}}=12.64 \mu \mathrm{m}\right)$, exhibiting largely improved quality factor (8140) and cleaner $\mathrm{S}_{11}$ spectrum.

\section{CONCLUSION}

To conclude, this paper presented the exploration of thick and high-quality aluminium nitride layers on sapphire for the fabrication of high quality factor SAW resonators in the 433 MHz ISM band. The high thermal stability of the aluminium 
nitride synchronous resonators has been proven up to $600^{\circ} \mathrm{C}$ using standard aluminium electrodes. Based on these first measurements, quasi-synchronous optimized resonators were designed, with a $Q$-factor higher than 8000 . This design was validated up to $400^{\circ} \mathrm{C}$, with quality factors remaining as high as 3400 at this temperature, which remains large enough for the future exploitation of the resonators as wireless temperature sensors. Meanwhile, the figure of merit shows a limited loss of $20 \%$ compared to room temperature.

The perturbations occurring at $450^{\circ} \mathrm{C}$ are the result of the larger sensitivity of the optimized design to the electrical behavior of the electrodes which encounters steady oxidation process, leading to a detuning of the resonators. This leads to a stronger, unrecoverable loss in the quality factor and in the figure of merit. The high temperature behavior of the complete resonator structure will then have to be taken into account in the optimization process.

Overall, this paper proves that it is possible to design wireless sensors based on AlN/Sapphire SAW resonators, which now opens new challenges for compatible electrodes. A trade-off needs to be found between their acoustic properties, and their chemical stability at high temperature. Once passivated, aluminium can still be considered for this purpose, in order to reach longer lifespans and larger temperatures for sustained use of SAW sensors based on aluminium nitride.

\section{REFERENCES}

[1] D. S. Ballantine, R. M. White, S. J. Martin, A. J. Ricco, E. T. Zellers, G. C. Frye, H. Wohltjen, Acoustic Wave Sensors. San Diego, CA, USA: Academic, 1997, p. 337.

[2] E. Born, J. Hornsteiner, T. Metzger, and E. Riha, "Diffusion of Niobium in Congruent Lithium Niobate," Phys. Stat. Sol. (a), vol. 177, pp. 393-400, Apr. 2000, doi: 10.1002/(SICI)1521-396X(200002)177:2<393::AID-

PSSA393>3.0.CO;2-F.

[3] R. Hauser, L. Reindl, and J. Biniasch, "Hightemperature stability of LiNbO3 based SAW devices," in Proc. 2003 IEEE Symp. on Ultr., Honolulu, HI, USA, 2003, vol. 1, pp. 192-195.

[4] M. P. Da Cunha, A. Maskay, R. J. Lad, D. J. Frankel, S. Moulzolf, M. Call, and G. Bernhardt, "Pt-Ni / Pt-Zr electrodes for stable SAW resonator operation during repeated temperature cycling up to $1000^{\circ} \mathrm{C}$," in Ultrasonics Symposium: Proc. 2015 IEEE Int. Ultr. Symp., Taipei, Taiwan, 2015, pp. 14.

[5] T. Kim, J. Kim, R. Dalmau, R. Schlesser, E. Preble and X. Jiang, "High-temperature electromechanical characterization of AIN single crystals," IEEE Trans. Ultr. Ferr. Freq. Contr, vol. 62, no. 10, pp.1880-1887, October 2015

[6] T. Aubert, J. Bardong, O. Legrani, O. Elmazria, B. Assouar, G. Bruckner, and A. Talbi, "In situ high-temperature characterization of AlN-based surface acoustic wave devices," J. Appl. Phys., vol. 114, 014505, Jul. 2013, doi: 10.1063/1.4812565.

[7] T. Aubert, O. Elmazria, B. Assouar, E. Blampain, A. Hamdan, D. Genève, and S. Weber, "Investigations on AlN/sapphire piezoelectric bilayer structure for hightemperature SAW applications," IEEE Trans. Ultr. Ferr. Freq. Contr., vol. 59, no. 5, pp.999-1005, May 2012, doi: 10.1109/TUFFC.2012.2285.

[8] O. Ambacher, M. S. Brandt, R. Dimitrov, T. Metzger, M. Stutzmann, R. A. Fischer, A. Miehr, A. Bergmaier, and G. Dollinger., "Thermal stability and desorption of Group III nitrides prepared by metal organic chemical vapor deposition," J. Vac. Sci. Tech. B, vol. 14, no. 6, pp. 3532-3542, Jun. 1996, doi: 10.1116/1.588793.

[9] S. Ballandras, V. Laude, T. Pastureaud, M. Wilm, W. Daniau, A. Reinhardt, W. Steichen and R. Lardat, "A FEA/BEM approach to simulate complex electrode structures devoted to guided elastic wave periodic transducers," IEEE International Ultrasonic Symposium 2002, Munich, Germany, Oct. 08-11 2002, pp.321-324,

[10] S. Ballandras, A. Reinhardt, V. Laude, A. Soufyane, S. Camou, W. Daniau, T. Pastureaud, W. Steichen, R. Lardat, M. Solal and P. Ventura, "Simulations of surface acoustic wave devices built on stratified media using a mixed finite element/boundary integral formulation," J. of Appl. Phys., vol. 96, no. 12, pp.7731-7741, 2004

[11] T. Pastureaud, R. Lardat, W. Steichen and P. Ventura, "Analysis of SAW propagation under a periodic multielectrode-type grating," IEEE International Ultrasonics Symposium 2005, Rotterdam, Netherlands, Sept 18-21 2005, pp.683-686

[12] B. François, J. M. Friedt, G. Martina, and S. Ballandras, "High temperature packaging for surface acoustic wave transducers acting as passive wireless sensors," Sensors and Actuators A: Phys., vol. 224, pp. 6-13, Apr. 2015, doi: 10.1016/j.sna.2014.12.034.

[13] P. V. Wright, "Analysis and Design of Low-Loss SAW Devices with Internal Reflections Using Coupling-of-Modes Theory," in Proc. IEEE Ultr. Symp., Montreal, Quebec, Canada, 1989, vol. 1, pp.141-152.

[14] J. Meltaus, V. P. Plessky, and S. S. Hong, "Nonsynchronous resonators on leaky substrates," in Proc. 2005 IEEE Ultr. Symp., Rotterdam, The Netherlands, 2005, pp. 21532156.

[15] C. Caliendo, "Theoretical and experimental investigation of gigahertz-band, temperature-compensated electromechanical coupling configurations based on AlN films," Appl. Phys. Lett., vol. 92, 033505, Jan. 2008, doi: 10.1063/1.2837179 
[16] B. Abdallah, C. Duquenne, M. P. Besland, E. Gautron, P. Y. Jouan, P. Y. Tessier, J. Brault, Y. Cordier, and M. A. Djouadi, "Thickness and substrate effects on AlN thin film growth at room temperature," Eur. Phys. J. - Appl. Phys., vol. 43, pp. 309-313, Sept. 2018, doi: 10.1051/epjap:2008082.

[17] C. Duquenne, M. A. Djouadi, P. Y. Tessier, P. Y. Jouan, M. P. Besland, C. Brylinski, R. Aubry, and S. Delage, "Epitaxial growth of aluminum nitride on $\mathrm{AlGaN}$ by reactive sputtering at low temperature," Appl. Phys. Lett., vol. 93, 052905, Aug. 2008, doi: 10.1063/1.2967816.

[18] M.B. Assouar, O. Elmazria, V. Mortet, P.-Y. Jouan, M. A. Djouadi, and P. Alnot, "Piezoelectric Aluminum Nitride Films Deposited by Triode Sputtering for Surface Acoustic Wave Devices," Ferroelectrics, vol. 273(1), pp. 249-254, 2002, 10.1080/00150190211764

[19] K. Ait Aissa, A. Achour, J. Camus, L. Le Brizoual, P.-Y. Jouan, and M.-A. Djouadi, "Comparison of the structural properties and residual stress of AlN films deposited by dc magnetron sputtering and high power impulse magnetron sputtering at different working pressures," Thin Solid Films, vol. 550, pp. 264-267, Jan. 2014, doi: 10.1016/j.tsf.2013.11.073.

[20] K. Ait Aissa, A. Achour, O. Elmazria, Q. Simon, M. Elhosni, P. Boulet, S. Robert, and M.-A. Djouadi, "AlN films deposited by dc magnetron sputtering and high power impulse magnetron sputtering for SAW applications," J. Phys. D: Appl. Phys., vol. 48, 145307, Mar. 2015, doi: 10.1088/0022$3727 / 48 / 14 / 145307$.

[21] F. Martin, P. Muralt, M.-A. Dubois, and A. Pezous, "Thickness dependence of the properties of highly c-axis textured AIN thin films," J. Vac. Sci. Technol. A, vol. 22(2), pp. 361-365, Feb. 2004, doi: 10.1116/1.1649343.

[22] K.-Y. Hashimoto, Surface Acoustic Wave Devices in Telecommunications. Berlin, Germany: Springer, 2000, p. 125.

[23] K. M. Lakin, "Equivalent Circuit Modeling of Stacked Crystal Filters," in $35^{\text {th }}$ Ann. Freq. Contr. Symp.: Proc. Ann. Freq. Contr. Symp., Philadelphia, USA, 1981, pp. 257-262.

[24] D. A. Feld, R. Parker, R. Ruby, P. Bradley, and S. Dong, "After 60 Years: A New Formula for Computing Quality Factor is Warranted," in Proc. 2008 IEEE Int. Ultr. Symp., Beijing, China, 2008, pp. 431-436.

[25] F. Bartoli, T. Aubert, M. Moutaouekkil, J. Streque, P. Pigeat, S. Zhgoon, A. Talbi, S. Hage-Ali, H. M'Jahed, and O. Elmazria, "AlN/GaN/Sapphire heterostructure for hightemperature packageless acoustic wave devices," Sensors and Actuators A: Phys., vol. 283, pp. 9-16, Nov. 2018, doi: 10.1016/j.sna.2018.08.011.

[26] W Daniau, T. Laroche and S. Ballandras, "Surface acoustic wave sensor that can be polled remotely," European patent
EP3032742A2

https://patents.google.com/patent/EP3032742A2/en

[27] A. Shvetsov, S. Zhgoon, I. Antcev, S. Bogoslovsky and G. Sapozhnikov, "Quartz Orientations for Optimal Power Efficiency in Wireless SAW Temperature Sensors, "in Proc. 2016 Eur. Freq. and Time Forum (EFTF), York, UK, 2016, pp. 1-4.

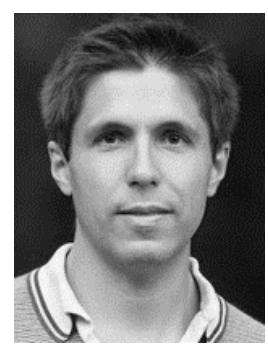

Jérémy Streque received the Ingénieur degree from École Centrale de Lille, France, and the M.S. degree from the Lille University of Science and Technology in 2007. He then received the Ph.D. degree from École Centrale de Lille in 2011, with a focus on magnetostatic actuation and soft materials for MEMS. He carried out his Ph.D. research at Institute of Electronics, Micro-electronics, and Nanotechnology. In 2012, he joined GeorgiaTech-CNRS, Metz, France, a Joint Laboratory focused on III-Nitrides for solar cells and sensors, and then worked for the launch of Institut Lafayette, Metz, a scientific private platform dedicated to these topics.

$\mathrm{He}$ is currently a researcher at Institut Jean Lamour, Nancy, France. His current areas of research are piezoelectric materials and SAW sensors for high temperature applications.

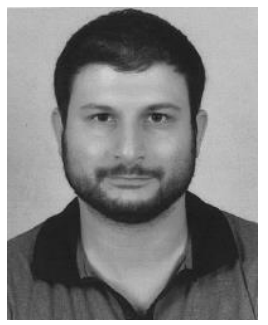

Sami Hage-Ali was born in Strasbourg, France, in 1982. He received an Engineering Degree from Ecole Centrale de Lille and a M.S in micronanotechnology from University of Lille 1 in 2005. He received another Master degree in international projects engineering from University of Lille 1 in 2006. He received a Ph.D. in micro-nanotechnology, acoustics and telecommunications in 2011. In 2011, Dr Hage-Ali was awarded a Fulbright grant and became a post-doctoral fellow at University of Illinois at Urbana-Champaign, USA.

Since 2014, he is an associate professor at University of Lorraine and is with the Micro-nanosystems group of Institut Jean Lamour, Nancy, France. Dr Hage-Ali's areas of research are flexible/stretchable electronics, micro-nanosystems, microwaves, antennas and surface acoustic wave sensors.

$\mathrm{He}$ is currently serving as chairman of the IEEE France Section Sensors Council Chapter. 


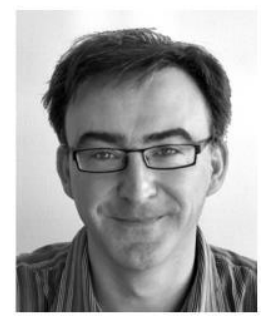

Thierry Aubert was born in Epinal, France, in 1975. In 1999, he passed the "Agrégation de Physique", a French national highly competitive exam. Then, he received the M.Sc. degree in Physics and the Ph.D. degree from the University of Nancy I, France, in 2007, and 2010 respectively. His Ph.D. thesis work was focused on the choice of materials for high temperature SAW applications. He studied in particular the possibility to use AlN/Sapphire bilayer structure for such aim. Between November 2010 and July 2011, he was with the CTR, Villach, Austria, where he obtained new results by achieving in situ electrical characterization of langasite and AlN-based SAW devices in high-temperature environment, up to temperatures above $1100^{\circ} \mathrm{C}$. He then has been an Associate Professor with the Universite de Savoie, Annecy, France. Since September 2014, he is with CentraleSupélec, a high ranking French Higher Education and Research Institution, in the LMOPS laboratory (Metz, France), of which he is deputy director since March 2017. His research activities are focused on the investigation of new materials and structures for high-temperature SAW applications (WLAW structures, Ir/Rh thin film electrodes, piezoelectric ScAIN thin films and stoichiometric lithium niobate crystals).

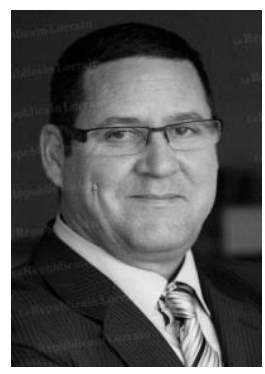

Hamid M'Jahed received his Master degree in electronics and industrial automation in 1993. He joined the Institut Jean Lamour as permanent CNRS (National Center for Scientific Research) staff.

Now he is CNRS engineer in RF and Electronics. His main research interests include study and realization of system query for SAW wireless sensors. In 2011, he receives the Crystal award as the best Engineer in CNRS.

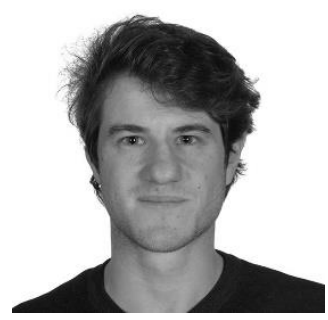

Mohammad Rammal received his Mechanical Engineering Degree from the Lebanese International University (LIU) in 2014. He also received a MSc in Thermal Sciences and Energy from École Polytechnique de l'Université de Nantes (Polytech Nantes) in 2015. He was involved in a European Project (TIPS) to pursue his $\mathrm{PhD}$ studies at the Institut des Matériaux de Nantes Jean Rouxel (IMN). He developed thermal management solutions for photonic devices: AlN deposition, characterization and integration. He earned his $\mathrm{PhD}$ degree in 2019. Later, he joined the Laboratoire de Thermique et d'Energie de Nantes CNRS (LTeN) to follow a postdoctoral research; the work targeted the measurement of thermal properties of rubber in order to generate heat in solids for sustainable energy applications.

Since 2020, Mohammad is working as a thermal engineer at Maana Electric in Luxembourg, designing and developing a thermal control system integrated in the production of solar panels without any greenhouse gas emissions.

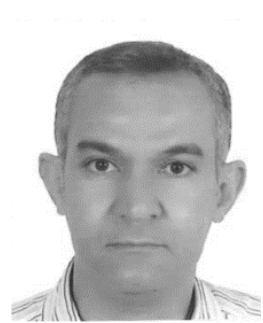

Omar Elmazria (M'2001-SM'16) received his $\mathrm{Ph} . \mathrm{D}$. degree in electronics from Metz University, France, in 1996 and he joined the University of Nancy I as associate Professor of electronic and communication systems in 1997 and as a full Professor in 2003.

$\mathrm{He}$ was the Head of the Micronanosystems group within the Institut Jean Lamour. His current research focus on layered structures-based surface acoustic waves (SAW) devices for communication systems and sensing applications.

Pr. Elmazria co-authored more than 150 papers in refereed international journals and in proceeding of international conferences. In 2017, he received the medal of URSI-France (International Union of Radio Science). 